\title{
A Hidden Threat: Subungual Melanoma in Hand
}

\author{
Chris Y. K. Tang, Boris Kwok-Keung Fung, C. P. Lung \\ Department of Orthopaedics and Traumatology, University of Hong Kong Medical Centre, \\ Queen Mary Hospital, Hong Kong (China) \\ Email: medic.chris.tang@gmail.com
}

Received November 16, 2011; revised December 26, 2011; accepted January 5, 2012

\begin{abstract}
Subungual melanoma, a relatively uncommon disease, has a worse prognosis than cutaneous melanoma in other sites. Both this life-threatening disease and its treatment cause cosmetic and functional impairment to the patient. The diagnosis is often delayed and resulting an advanced disease at presentation. The important clinical manifestation, diagnosis and latest treatments for subungual melanoma are reviewed. Electronic databases of Medline, PubMed, and the Cochrane library were searched with the key word "subungual melanoma". A clinical scoring system is proposed by the authors to stratify patients to undergo biopsy, making earlier diagnosis of this disease possible. The latest trend of treatment involves wide local excision at a more distal level of the affected digit, in contrast to radical amputation of the digit in the past. Studies have shown that this does not decrease the survival nor increase local recurrence, but reduces the functional impairment for the patient. This review aims to summarise the approach to subungual melanoma for clinicians, so that they can recognise and diagnose this disease early and generate the best functional and cosmetic outcome for the patients.
\end{abstract}

Keywords: Subungual Melanoma; Hand

\section{Introduction}

Subungual melanoma is a relatively uncommon disease, accounting for $1 \%$ to $3 \%$ of all cutaneous melanoma in Caucasians [1], with an estimate in 2010 that 1 of every 39 people in the western countries will be diagnosed with cutaneous melanoma during his or her lifetime [2]. In contrast to melanoma in other sites, it has a worse prognosis, with the 5-year survival rate ranges from $16 \%$ to $87 \%[3,4]$. Histological diagnosis is frequently delayed due to clinical misdiagnosis. There is a mean delay of 9 months in $50 \%$ of cases [5]. The mean Breslow thickness at diagnosis is $4.8 \mathrm{~mm}$ [6], and 79\% are found as Clark level IV (invaded to lower dermis) [7], signifying advanced disease at diagnosis when comparing to cutaneous melanoma. Cutaneous melanoma with Breslow thickness of less than $1.5 \mathrm{~mm}$ is normally consistent with a high 5-year survival rate [8], but the cutoff for subungual melanoma remains to be elucidated by further research. Despite the importance of subungual melanoma, there is lack of systematic review on this topic in recent years. This review article serves to summarise the important aspects of subungual melanoma.

\section{Materials and Methods}

Electronic databases of Medline, PubMed, and the Cochrane library were searched. Articles written in English were included. Epidemiology, etiology, clinical manifestations, diagnosis and treatment were reviewed. References in review articles were screened for potentially relevant studies not yet identified.

\section{Etiology}

Ultraviolet (UV) radiation exposure has been a wellknown risk factor for cutaneous melanoma. Yet significant UV radiation is unlikely to penetrate the nail plate to cause subungual melanoma [9]. 23\% - 44\% of patients report a relationship between direct trauma and the onset of subungual melanoma, but whether it is related to trauma remains controversial $[7,10,11]$. The relationship can be explained by coincidence, increased awareness to the dark area under the nail, bleeding of subclinical subungual melanoma caused by the trauma or trauma-induced melanocyte mutation [12].

\section{Clinical Manifestations}

The mean age of presentation is 55.4 years old, with men more than women [13]. The most common presentation is discoloration of nail, others include a non-healing wound, a mass, a split in the nail and bleeding [14]. Eyal K. Levit (2000) summarized an ABCDEF approach to examine subungual melanoma [15] (See Table 1).

The peak incidence for subungual melanoma is in the 
Table 1. Approach to examine subungual melanoma.

A

Age: Range 20 - 90 y, peak 5th-7th decades

Race: African-American, native American, Asian

Band (nail band)

B

Pigment of band (Brown-Black)

Breadth of band (more than $3 \mathrm{~mm}$ )

Border of band (irregular/blurred)

Change: rapid increase in size/growth rate of nail band

C Lack of Change: Failure of nail dystrophy to improve despite adequate treatment

Digit involved: Thumb > index finger

D Single digit $>$ multiple digits

Dominant hand

E

Extension: Extension of pigment to involve proximal or lateral nail fold (Hutchinson's sign) or free edge of nail plate

F

Family or personal history: of previous melanoma or dysplastic nevus syndrome

50 - 70 years old, while the benign pigmented bands, which are often melanotic macule histologically, are more likely in the younger patients $[10,16]$. The disease is more common in Asian and African than in Caucasian. Pigmentation in the nail band (Figure 1) also raises the suspicion. However, in comparison to $7 \%$ of cutaneous melanoma, amelanotic melanoma contributed to $37 \%$ $(14 / 38)$ in one of the series [17]. Absence of pigmentation therefore does not exclude subungual melanoma. Nail band more than $3 \mathrm{~mm}$ should be suspected for subungual melanoma. The cutoff of $3 \mathrm{~mm}$ is arbitrary and is related to the growth pattern of melanoma: the lesion has grown to more than $3 \mathrm{~mm}$ when the melanoma is still in-situ, with the nests of melanocytes within the epidermis [18]. Recent and acute increase in size is suggestive of melanoma [19]. Subungual melanoma in hand is found most commonly in the thumb (58\% (22/39)) which is the most crucial digit for hand function [17]. Subungual melanoma is more common in the hands than in the feet with a ratio of approximately $3: 2$ [10,13]. It occurs most often in the thumb and great toe. There has not been any evidence to suggest the cause of this discrepancy, but some authors suggest that this may be due to increased sun exposure to the hand during outdoor work and activities (particularly the thumb during grip) [13]. Alternatively, this could be due to a higher chance of trauma in the hand than in the foot.

Subungual melanoma also presents as irregular nail band which is usually wider than $3 \mathrm{~mm}$, heterogeneous in colour and not crisp at its lateral margins. With disease progression, there is involvement of nail bed or periun-

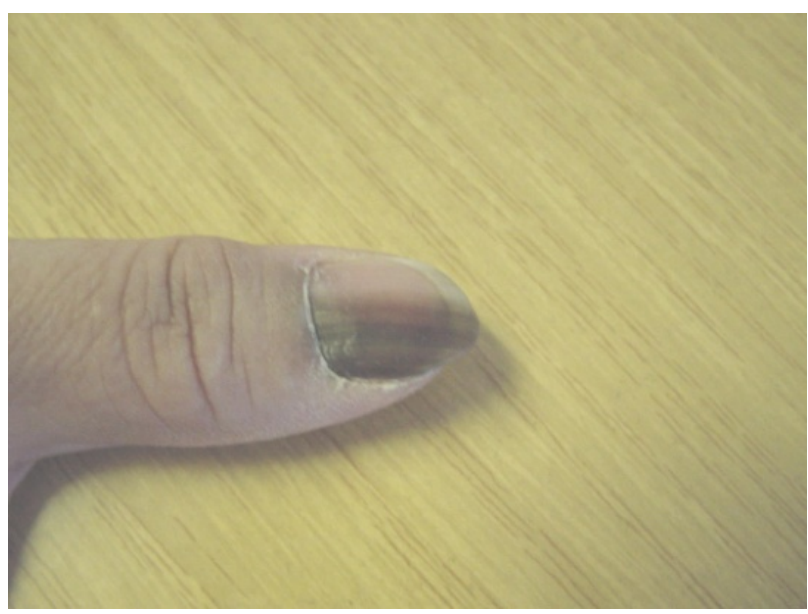

Figure 1. Pigmented nail band in subungual melanoma.

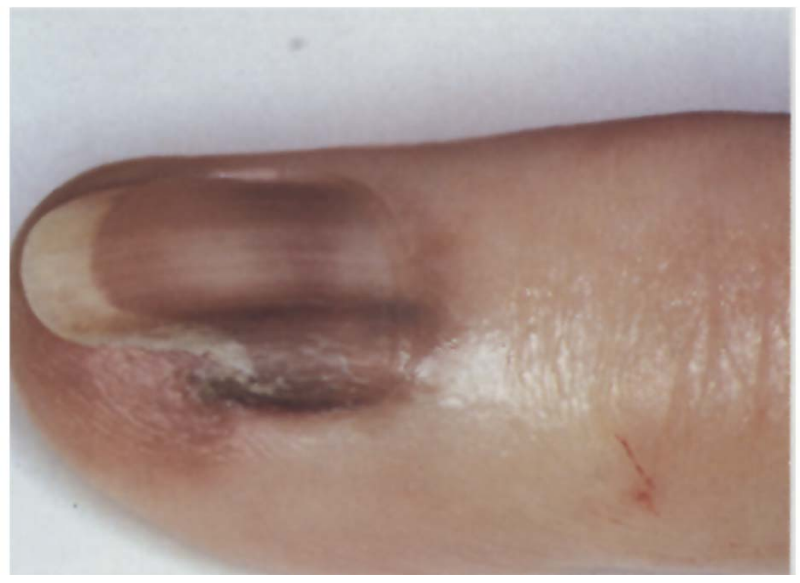

Figure 2. Hutchinson's sign in a subungual melanoma. Note the pigmented nail band and extension of pigmentation to proximal nailfold. (Courtesy of American Academy of Dermatology, Inc Hutchinson's sign: A reappraisal. 1996.)

gual tissue, resulting increased size and irregularity of the band. Hutchinson's sign (periungual extension of pigment into proximal and/or lateral nailfolds) (Figure 2) is traditionally regarded as an important sign. However, it is not pathognomonic [20]. This is best regarded as a clinical sign when there is subungual melanoma confirmed histologycally, since a number of other conditions mimic Hutchinson's sign, including subungual haematoma, regressing nevoid melanosis in childhood, ethinic pigmentation, Laugier-Hunziker syndrome, Peutz-Jegher syndrome, radiation therapy, malnutrition, minocycline. Moreover, it is often a late sign. Concomitant thinning or fissuring of nail plate with abnormal pigmentation is another suggestive feature. With further progression, a nodule may develop. Ulceration and nail plate destruction can occur eventually [21].

Patients with personal or family history of dysplastic melanocytic naevi have a higher risk for melanoma. Surveillence by photography is cost-effective [22]. 


\section{Diagnosis}

Biopsy of the lesion remains the gold standard for diagnosis. It is difficult to diagnose subungual melanoma in the early stages, due to its relatively low incidence and the difficulty in making a clinical diagnosis. The authors propose a clinical scoring system to stratify the patients to undergo biopsy as the following (See Table 2).

The total score of $0-2,3-5$, more than 5 represents low risk, medium risk and high risk group respectively. These three categories serve to stratify patients to undergo nail biopsy for investigation. Although Hutchinson's sign may represent some benign conditions as mentioned above, it is worth to perform a biopsy as this may represent advanced subungual melanoma. In subungual haematoma, most of them will grow along the nail with obvious movement over 4 - 6 weeks, with a depigmented area appearing in the region of lunula [5]. However, this does not occur in subungual melanoma. It is recommended biopsy should be performed in the medium risk and high risk groups. The authors believe that this scoring system can help clinicians select higher risk patients for biopsy, making earlier diagnosis possible. Owing to the uncommon occurrence of this disease, the exact sensitivity and specificity of this scoring system remain to be validated in further studies.

In very early stage of subungual melanoma, it is often misdiagnosed as benign conditions such as benign melanonychia striata, melanocytic nevus or "no pathological diagnosis". This is because the atypical melanocytes have subtle degree of atypia and mitosis that cannot be detected due to their small sizes. Features favour subungual melanoma includes "skip lesion" (proliferation of atypical melanocytes in hyponychium is greater than that from nail matrix) and histological confirmed Hutchinson's sign [23].

A good biopsy (Figure 3 \& Figure 4) consists of an incisional biopsy should be taken from the matrix origin

Table 2. Proposed clinical scoring system.

\begin{tabular}{lc}
\hline Risk Factors & Score \\
\hline 50 - 70 years old & 1 \\
Ethnic origin: Asian, African & 1 \\
Pigmented nail band (Brown-Black) & 2 \\
Breadth of band (more than 3 mm) & 2 \\
Border of band (irregular/blurred) & 2 \\
Change: rapid increase in size/growth rate of nail band & 2 \\
Thumb involvement & 2 \\
Hutchinson's sign & 3 \\
No change after 4 - 6 weeks of observation & 3 \\
\hline
\end{tabular}

Total score: low risk: 0 - 2, medium risk: 3 - 5, high risk: $>5$.

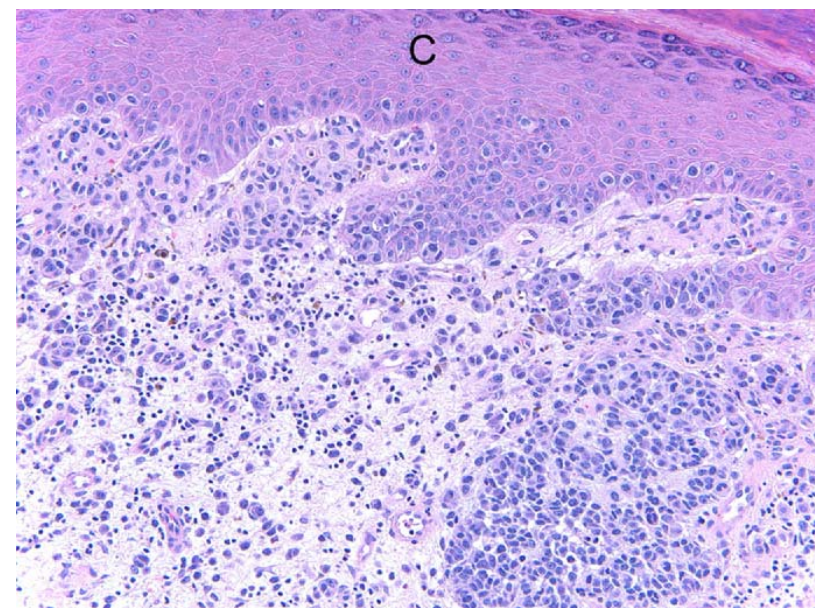

Figure 3. Subungual melanoma. Atypical melanocytes arrayed irregularly within the nail bed epithelium and within the dermis, without maturation with descent. (Courtesy of Pigmented Lesions of the Nail Unit. Clinical and Histopathologic Features. Beth. 2010).

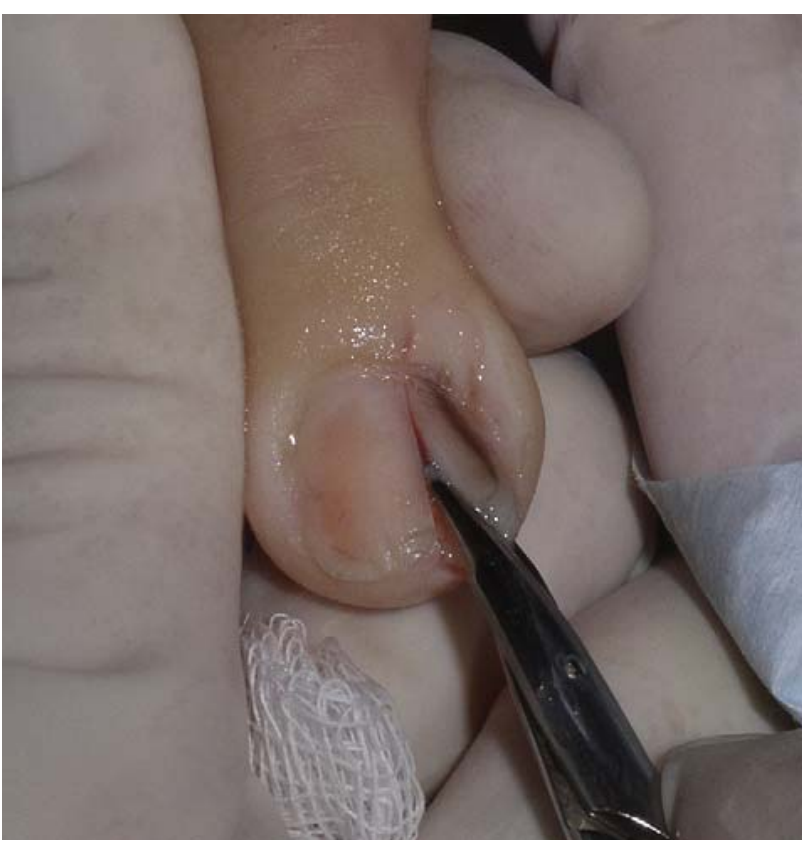

Figure 4. Nail biopsy. (Courtesy of Nathaniel Jellinek. Nail matrix biopsy of longitudinal melanonychia: Diagnostic algorithm including the matrix shave biopsy. Journal of the American Academy of Dermatology. 2007).

of the pigmented nail band, in addition to the nail bed. A $2 \mathrm{~mm}$ punch is usually used to perform the biopsy of an adequate amount of lesion, without causing nail plate dystrophy. Retraction of nail plate before biopsy can also strip the epithelium from dermal part of the specimen [24]. Subungual melanoma in-situ is often seen at the peripheral edges of subungual melanoma. Features suggestive of subungual melanoma include architecture of the cells (increased number of irregularly distributed melanocytes along basal layer of nail matrix), nuclear 
morphology and host reaction (lymphocytic infiltrates). As a result, incomplete biopsy of the lesion will make the diagnosis of subungual melanoma in-situ instead of subungual melanoma, impairing the accuracy of the pathological diagnosis. Further biopsy should be obtained if histopathological features are not consistent with clinical features [25].

The American Joint Commission on Cancer (AJCC) revised the staging for melanoma in 2010, major changes included 1) for patients with localized (stage I or II) melanoma, tumor thickness, mitotic index and ulceration are considered the most powerful prognostic parameters; 2) mitotic index was used instead of Clark level of invasion for patients with thin primary tumors; 3) all patients with microscopic melanoma metastases were classified as stage III; 4) for patients with regional lymph node metastases, the number of lymph nodes involved, metastatic tumor burden and ulceration and thickness of the primary tumor were the most predictive independent factors of survival and 5) for patients with distant metastases, the site (non-visceral vs. lung vs. other visceral) and serum lactate dehydrogenase elevation contributed the $\mathrm{M}$ category [26].

\section{Treatment}

The mainstay of treatment is surgical amputation. Covering of the defect by skin graft is only needed after surgical excision. Traditionally since 1886, radical surgical amputation at the level of metacarpal bones has been used [27]. However, this resulted in an impaired function and cosmesis. Researchers tried to move the amputation level more distally, aiming at a better residual function without compromising the survival and local control. In 1992-1994, two studies found that conservative amputation of affected digit at the level of proximal interphalangeal joint with clear margin makes no difference in patient survival as compared with those having more proximal amputations $[28,29]$. Elective lymph node dissection [30,31] and regional limb perfusion does not affect patient survival, but may have a role in reducing local recurrence from $27 \%$ to $20 \%$ [28,29]. Finley [32] later reported similar survival and recurrence when comparing amputation at distal interphalangeal joint and more proximal amputation. Yet the distal interphalangeal joint amputation group contained only 4 patients out of the 22 patients. Moehrle [33] in Germany reported a "functional” surgery for subungual melanoma, in which the tumour was surgically removed by a wide local excision with safety margin of $10 \mathrm{~mm}$ and a partial resection of distal phalanx. They concluded that the more distal amputations of subungual melanoma of hand do not compromise survival nor increase local recurrence. However, there was selection bias, because allocation to the "functional” surgery group was done after having histopa- thological results from initial excision. Many of this kind of wide local excision are prone to have local recurrence or persistent positive margins, requiring eventual amputation [14]. Mohs micrographic surgery is a technique of excision of the primary subungual melanoma, with complete microscopic evaluation of the lateral and deep margins. It can reduce the amount of tissue resected. It yields a $79 \%$ disease-free survival (with a mean follow-up of 7.7 years) but a higher rate of marginal recurrence. Further studies regarding this technique is needed.

There is no study done for the excision margin specifically for subungual melanoma, but there are a few for cutaneous melanoma. Veronesi [34] compared $1 \mathrm{~cm}$ with $3 \mathrm{~cm}$ or more excision margins and found similar disease-free survival and overall survival, thus suggesting a 1 $\mathrm{cm}$ excision margin for lesions $<1 \mathrm{~mm}$ in depth. Balch [35] compared $2 \mathrm{~cm}$ with $4 \mathrm{~cm}$ excision margins and found similar rates of 10-year survival and local recurrence, thus suggesting a $2 \mathrm{~cm}$ excision margin for lesions of $1 \mathrm{~mm}-4 \mathrm{~mm}$ in depth. Thomas [36] compared $1 \mathrm{~cm}$ with $3 \mathrm{~cm}$ excision margins and found similar overall survival rates, but there is a higher local recurrence rate in 1-cm excision margin group. They therefore suggested 3-cm excision margin for lesions of $2 \mathrm{~mm}$ or more in depth.

A randomized controlled showed that sentinel lymph node biopsy has staging and prognostic implication for melanoma with intermediate thickness $(1.2$ to $3.5 \mathrm{~mm})$. Immediate lymphadenectomy in cases of positive sentinel lymph node biopsy improves disease-free survival [37].

Six prognostic factors are related to overall survival: stage at presentation, presence of ulceration, tumour thickness, presence of bone invasion, aneuploid fraction and S-phase fraction [28].

Since melanoma is an immunogenic tumour, immunotherapy including high-dose interleukin-2 in metastatic disease, alpha-interferon for resected high-risk nodal disease and ipilimumab (cytotoxic T lymphocyte antigen-4 (CTLA-4) blocking antibody) are useful [38].

\section{Discussion}

Subungual melanoma is a relatively uncommon disease. Although some of the studies came from cutaneous melanoma, there is no evidence suggests that subungual melanoma behaves differently, and the worse prognosis subungual melanoma can be explained by the delayed diagnosis. Although some studies postulated trauma as the etiology, its role remains to be further elucidated. Important clinical presentations include older age group (when compare to other benign conditions), pigmented nail band more than $3 \mathrm{~mm}$, rapid increase in size, thumb involvement, Hutchinson's sign.

The authors propose a clinical scoring system to strati- 
fy patients to undergo biopsy and recommend biopsy to be done in the medium and high risk groups. It should be aware that there are pitfalls in the histopathological diagnosis of subungual melanoma. The pitfalls include misdiagnosing very early stage subungual melanoma as other benign conditions ("skip lesion" and histological confirmed Hutchinson's sign favour the diagnosis of subungual melanoma) and poor technique of taking a nail biopsy including underestimation of subungual melanoma as subungual melanoma in-situ.

Treatment of subungual melanoma has been revolutionized as compared to that in Hutchinson's era. Modern trend involves moving the level of excision more distally, yielding a better residual hand function, without compromising the survival and local control. The treatment evolves from traditional radical amputation of the digit, to excision at the level of proximal interphalangeal joint, distal interphalangeal joint, to wide local exicison. Mohs micrographic surgery is a further advance but more studies need to be carried out to identify its role.

Nevertheless, all these advances in treatment make no difference if the diagnosis of subungual melanoma is delayed. We have to go back to traditional radical excision of the digit for the advanced disease, resulting a great adverse impact on the patient functionally and cosmetically. Early diagnosis means better residual function, and delayed diagnosis leads to the worse prognosis in subungual melanoma. Although this disease is rare, it is a rare disease too important for us to miss. In order to tackle the problem of delayed diagnosis, the authors proposed a clinical scoring system to stratify patients to undergo biopsy. As a result, earlier diagnosis could be made from the nail biopsy, and proper treatment can be initiated promptly. Future direction of study involves validation of the proposed clinical scoring system.

Finally, it is hoped that with this review, there will be more attention from the clinicians for this uncommon but potentially functional debilitating disease, allowing the traditional radical excision of the digit to be a history for subungual melanoma treatment.

\section{REFERENCES}

[1] K. Blessing, N. M. Kernohan and K. G. M. Park, "Subungual Malignant Melanoma: Clinicopathological Features of 100 Cases,” Histopathology, Vol. 19, No. 5, 1991, pp. 425-429. doi:10.1111/j.1365-2559.1991.tb00232.x

[2] A. I. Riker, N. Zea and T. Trinh, “The Epidemiology, Prevention, and Detection of Melanoma," The Ochsner Journal, Vol. 10, 2010, pp. 56-65.

[3] Banfield and Dawber, "Nail Melanoma: A Review of the Literature with Recommendations to Improve Patient Management,” British Journal of Dermatology, Vol. 141, No. 4, 1999, pp. 628-632. doi:10.1046/j.1365-2133.1999.03099.x
[4] K. E. Thai, R. Young and S. Rd, "Nail Apparatus Melanoma," Australasian Journal of Dermatology, Vol. 42, No. 2, 2001, pp. 71-83.

[5] V. K. Shukla and L. E. Hughes, "Differential Diagnosis of Subungual Melanoma from a Surgical Point of View," British Journal of Surgery, Vol. 76, No. 11, 1989, pp. 1156-1160. doi:10.1002/bjs.1800761115

[6] Banfield, Redburn and Dawber, "The Incidence and Prognosis of Nail Apparatus Melanoma. A Retrospective Study of 105 Patients in Four English Regions,” British Journal of Dermatology, Vol. 139, No. 2, 1998, pp. 276279. doi:10.1046/j.1365-2133.1998.02365.x

[7] R. H. Patterson and E. B. Helwig, "Subungual Malignant Melanoma: A Clinical-Pathologic Study,” Cancer, Vol. 46, No. 9, 1980, pp. 2074-2087.

doi:10.1002/1097-0142(19801101)46:9<2074::AID-CNC R2820460928>3.0.CO;2-K

[8] C. M. Balch, S. J. Soong, et al., “An Analysis of Prognostic Factors in 8500 Patients with Cutaneous Melanoma,” In: C. M. Balch, A. N. Houghton, G. W. Milton, A. J. Sober and S.-J. Soong, Eds., Cutaneous Melanoma, Lippincott, Philadelphia, 1992, pp. 165-187.

[9] W. A. G. Bruls, H. Slaper, J. C. Van Der Leun and L. Berrens, "Transmission of Human Epidermis and Stratum Corneum as a Function of Thickness in the Ultraviolet and Visible Wavelengths," Photochemistry and Photobiology, Vol. 40, No. 4, 1984, pp. 485-494. doi:10.1111/j.1751-1097.1984.tb04622.x

[10] H. Takematsu, M. Obata, Y. Tomita, T. Kato, M. Takahashi and R. Abe, "Subungual Melanoma: A Clinicopathologic Study of 16 Japanese Cases,” Cancer, Vol. 55, No. 11, 1985, pp. 2725-2731. doi:10.1002/1097-0142(19850601)55:11<2725::AID-CN CR2820551134>3.0.CO;2-V

[11] D. A. Hudson, J. E. J. Krige, R. M. Strover and H. S. King, "Subungual Melanoma of the Hand," Journal of Hand Surgery, Vol. 15, No. 3, 1990, pp. 288-290. doi:10.1016/0266-7681(90)90005-O

[12] M. Möhrle and H. M. Häfner, "Is Subungual Melanoma Related to Trauma?” Dermatology, Vol. 204, No. 4, 2002, pp. 259-261. doi:10.1159/000063354

[13] J. A. O’Leary, K. R. Berend, J. L. Johnson, L. S. Levin and H. F. Seigler, "Subungual Melanoma. A Review of 93 Cases with Identification of Prognostic Variables," Clinical Orthopaedics and Related Research, Vol. 378, 2000, pp. 206-212.

[14] T. Cohen, K. J. Busam, A. Patel and M. S. Brady, "Subungual Melanoma: Management Considerations,” American Journal of Surgery, Vol. 195, No. 2, 2008, pp. 244248. doi:10.1016/j.amjsurg.2007.03.010

[15] E. K. Levit, M. H. Kagen, R. K. Scher, M. Grossman and E Altman, "The ABC Rule for Clinical Detection of Subungual Melanoma," Journal of the American Academy of Dermatology, Vol. 42, No. 2, 2000, pp. 269-274.

[16] R. K. Scher and D. N. Silvers, "Longitudinal Melanonychia Striata," Journal of the American Academy of Dermatology, Vol. 24, No. 6, 1991, pp. 1035-1036. doi:10.1016/S0190-9622(08)80136-3 
[17] M. J. Quinn, J. E. Thompson, K. Crotty, W. H. McCarthy and A. S. Coates, "Subungual Melanoma of the Hand," The Journal of Hand Surgery, Vol. 21, No. 3, 1996, pp. 506-511. doi:10.1016/S0363-5023(96)80371-6

[18] A. B. Ackerman, "Conventional Microscopy: Signs That Stamp Pigmented Melanocytic Nevi as Benign,” American Journal of Dermatopathology, Vol. 4, No. 5, 1982, pp. 461-466. doi:10.1097/00000372-198210000-00015

[19] T. Kato, Y. Usuba, H. Takematsu, N. Kumasaka, Y. Tanita, K. Hashimoto, Y. Tomita and H. Tagami, "A Rapidly Growing Pigmented Nail Streak Resulting in Diffuse Melanosis of the Nail. A Possible Sign of Subungual Melanoma in situ," Cancer, Vol. 64, No. 10, 1989, pp. 2191-2197.

doi:10.1002/1097-0142(19891115)64:10<2191::AID-CN CR2820641035>3.0.CO;2-D

[20] R. Baran and P. Kechijian, “Hutchinson's Sign: A Reappraisal," Journal of the American Academy of Dermatology, Vol. 34, No. 1, 1996, pp. 87-90. doi:10.1016/S0190-9622(96)90839-7

[21] B. S. Ruben, "Pigmented Lesions of the Nail Unit: Clinical and Histopathologic Features," Seminars in Cutaneous Medicine and Surgery, Vol. 29, No. 3, 2010, pp. 148158. doi:10.1016/j.sder.2010.06.008

[22] J. W. Kelly, J. M. Yeatman, C. Regalia, G. Mason and H. Ap, "A High Incidence of Melanoma Found in Patients with Multiple Dysplastic Naevi by Photographic Surveillance,” Medical Journal of Australia, Vol. 167, 1997, pp. 191-194.

[23] M. Izumi, K. Ohara, T. Hoashi, H. Nakayama, C.-S. Chiu, T. Nagai, J. Matsubayashi, K. Iwaya and K. Mukai, "Subungual Melanoma: Histological Examination of 50 Cases from Early Stage to Bone Invasion,” The Journal of Dermatology, Vol. 35, No. 11, 2008, pp. 695-703. doi:10.1111/j.1346-8138.2008.00551.x

[24] G. Massi, P. E. Leboit and E. Al, "Melanoma on Palms, Soles and Subungual Melanoma-Acral Melanoma,” In: D. Massi and P. Leboit, Eds., Histological Diagnosis of Nevi and Melanoma, Springer, 2004, p. 577. doi:10.1007/978-3-7985-1943-5

[25] R. A. Scolyer, J. F. Thompson, S. W. McCarthy, G. M. Strutton and D. E. Elder, "Incomplete Biopsy of Melanocytic Lesions Can Impair the Accuracy of Pathological Diagnosis," Australasian Journal of Dermatology, Vol. 47, No. 1, 2006, pp. 71-73.

[26] A. Piris, M. C. Mihm Jr. and L. M. Duncan, “AJCC Melanoma Staging Update: Impact on Dermatopathology Practice and Patient Management," Journal of Cutaneous Pathology, Vol. 38, No. 5, 2011, pp. 394-400. doi:10.1111/j.1600-0560.2011.01699.x

[27] J. Hutchinson, "Melanosis Often Not Black: Melanotic Whitlow,” British Medical Journal, Vol. 1, 1886, p. 491.

[28] K. M. Heaton, A. El-Naggar, L. G. Ensign, M. I. Ross and B. Cm, "Surgical Management and Prognostic Factors in Patients with Subungual Melanoma," Annals of
Surgery, Vol. 219, No. 2, 1994, pp. 197-204. doi:10.1097/00000658-199402000-00012

[29] K. G. M. Park, K. Blessing and K. Nm, "Surgical Aspects of Subungual Malignant Melanomas,” Annals of Surgery, Vol. 216, 1992, pp. 692-695. doi:10.1097/00000658-199212000-00012

[30] F. H. Sim, W. F. Taylor, D. J. Pritchard and S. Eh, "Lymphadenectomy in the Management of Stage I Malignant Melanoma: A Prospective Randomized Study,” Mayo Clinic Proceedings, Vol. 61, 1986, pp. 697-705.

[31] N. Cascinelli, A. Morabito, M. Santinami, R. M. Mackie and F. Belli, "Immediate or Delayed Dissection of Regional Nodes in Patients with Melanoma of the Trunk: A Randomised Trial,” Lancet, Vol. 351, No. 9105, 1998, pp. 793-796. doi:10.1016/S0140-6736(97)08260-3

[32] R. K. Finley 3rd, D. L. Driscoll, L. E. Blumenson and K. Cp, "Subungual Melanoma: An Eighteen-Year Review," Surgery, Vol. 116, 1994, pp. 96-100.

[33] M. Moehrle, S. Metzger, W. Schippert, C. Garbe, G. Rassner and H. Breuninger, “'Functional' Surgery in Subungual Melanoma,” Dermatologic Surgery, Vol. 29, 4, 2003, pp. 366-374. doi:10.1046/j.1524-4725.2003.29087.x

[34] U. Veronesi, N. Cascinelli, J. Adamus, C. Balch, D. Bandiera, A. Barchuk, R. Bufalino, P. Craig, J. De Marsillac, J. C. Durand and E. Al, “Thin Stage I Primary Cutaneous Malignant Melanoma. Comparison of Excision with Margins of 1 or $3 \mathrm{~cm}$," New England Journal of Medicine, Vol. 318, 1988, pp. 1159-1162. doi:10.1056/NEJM198805053181804

[35] C. M. Balch, S.-J. Soong, T. Smith, M. I. Ross, M. M. Urist, C. P. Karakousis, W. J. Temple, M. C. Mihm, R. L. Barnhill, W. R. Jewell, et al., "Long-Term Results of a Prospective Surgical Trial Comparing $2 \mathrm{~cm}$ vs. $4 \mathrm{~cm} \mathrm{Ex-}$ cision Margins for 740 Patients with 1 - 4 mm Melanomas,” Annals of Surgical Oncology, Vol. 8, No. 2, 2001, pp. 101-108.

[36] J. M. Thomas, J. Newton-Bishop, R. A'hern, G. Coombes, M. Timmons, J. Evans, M. Cook, J. Theaker, M. Fallowfield, T. O’neill, W. Ruka and J. M. Biss, "Excision Margins in High-Risk Malignant Melanoma,” New England Journal of Medicine, Vol. 350, 2004, pp. 757-766. doi:10.1056/NEJMoa030681

[37] D. L. Morton, J. F. Thompson, A. J. Cochran, N. Mozzillo, R. Elashoff, R. Essner, O. E. Nieweg, D. F. Roses, H. J. Hoekstra, C. P. Karakousis, D. S. Reintgen, B. J. Coventry, E. C. Glass and H. J. Wang, "SentinelNode Biopsy or Nodal Observation in Melanoma," New England Journal of Medicine, Vol. 355, 2006, pp. 13071317.

[38] J. Weber, "Immunotherapy for Melanoma," Current Opinion in Oncology, Vol. 23, 2011, pp. 163-169. doi:10.1097/CCO.0b013e3283436e79 